2012, August 14

KEK-TH-1562

\title{
SL(5) duality from canonical M2-brane
}

\author{
Machiko Hatsuda $^{\dagger * a}$ and Kiyoshi Kamimura ${ }^{\star b}$ \\ ${ }^{\dagger}$ Physics Department, Juntendo University, 270-1695, Japan \\ *KEK Theory Center, High Energy Accelerator Research Organization, \\ Tsukuba, Ibaraki 305-0801, Japan \\ ${ }^{\star}$ Department of Physics, Toho University, Funabashi, 274-8510, Japan \\ ${ }^{a}$ mhatsuda@post.kek.jp ; ${ }^{b}$ kamimura@ph.sci.toho-u.ac.jp
}

\begin{abstract}
We show how the SL(5) duality in M-theory is explained from a canonical analysis of M2-brane mechanics. Diffeomorphism constraints for a M2-brane coupled to supergravity background in $d=4$ are reformulated in a SL(5) covariant form, in which spatial diffeomorphism constraints are recast into a $\mathrm{SL}(5)$ vector and the generalized metric in the Hamiltonian constraint is quartic in the SL(5) generalized vielbein. The Hamiltonian for a M2 brane has the SL(5) duality symmetry in a background dependent gauge.
\end{abstract}




\section{Introduction}

In contrast to the pure gravity theory, string gravity and membrane gravity theories contain rich duality symmetries governed by generalized geometry introduced by Hitchin [1], Gualtieri [2] for string system and Hull [3] for M theory. For these theories the general coordinate transformation is generalized to the gauge transformation for both gravity field and gauge fields coupled to extended objects and it is given by Courant bracket or C-bracket $[4,5]$. Doubled formalism with manifest T-duality $[6,7]$ and double field theory formulated by [8] are complementary approaches to understand duality. Further studies appeared in $[9,10,11,12,13,14,15,16,17,18]$ and $[19,20,21,22,23,24]$ on D-branes.

For a bosonic string theory the gravitational field $G_{m n}$ and the rank two tensor $B_{m n}$ field are mixed by the T-duality symmetry, where the relation between T-duality in the first quantized level and the one in the second quantized level is well known. On the other hand U-duality in the first quantized level and the one in M-theory have not been fully understood so far. The U-duality, as the symmetry of solitonic charges associated with extended objects [25], is the hidden symmetry in the 11-dimensional supergravity theory [26]. The relation between the U-duality and a membrane duality was shown by Duff and Lu [27] using with the Gaillard-Zumino's (GZ) dual formulation [28]. Recently the supergravity is reformulated in a manifest duality covariant way by Berman and Perry [14], relating to the membrane duality which is also treated in the GZ dual formulation.

In this paper we examine the membrane duality in canonical language instead of the GZ dual formulation. We focus on the SL(5) duality for the case of $d=4$. In reference [23] one of the present author has made a canonical analysis of D-brane mechanics to obtain Courant brackets by using which the general coordinate transformation and the gauge transformation for D-branes are derived. In this paper we apply the above analysis to a M2 brane, and we clarify the relation between SL(5) duality in M2 mechanics and the one in the supergravity theory.

One of the typical features of actions of extended objects is the diffeomorphism invariance. For a string case the $\sigma$-diffeomorphism constraint, which corresponds to $\triangle=\frac{\partial}{\partial x^{m}} \frac{\partial}{\partial \tilde{x}_{m}}=0$ constraint for example in [8], plays an essential role. Diffeomorphism constraints for a bosonic string are written in terms of $Z_{M}=\left(p_{m}, \partial_{\sigma} x^{m}\right)$ which is the basis of the generalized geometry, where $x^{m}$ and $p_{m}$ are string coordinate and its conjugate momentum with $m=1, \cdots, d$. The $\sigma$-diffeomorphism constraint $\mathcal{H}_{\sigma}$ and the $\tau$-diffeomorphism constraint, Hamiltonian $\mathcal{H}_{\perp}$, for 
a string are written as

$$
\left\{\begin{array}{l}
\mathcal{H}_{\sigma}=\frac{1}{2} Z_{M} \rho^{M N} Z_{N}=0 \quad, \rho^{M N}=\left(\begin{array}{cc}
0 & \delta_{m}^{n} \\
\delta_{n}^{m} & 0
\end{array}\right) \\
\mathcal{H}_{\perp}=\frac{1}{2} Z_{M} \mathcal{M}^{M N} Z_{N}=0 \quad, \quad \mathcal{M}^{M N}=\left(\begin{array}{cc}
G^{m n} & -G^{m q} B_{q n} \\
B_{m p} G^{p n} & G_{m n}-B_{m p} G^{p q} B_{q n}
\end{array}\right)
\end{array}\right.
$$

where $\mathcal{M}^{M N}$ is the generalized metric as a function of $G_{m n}$ and $B_{m n}$. The $\sigma$-diffeomorphism constraint $\mathcal{H}_{\sigma}$ has $\mathrm{O}(d, d)$ invariance. Under the $\mathrm{O}(d, d) \ni g$ transformation, $Z \rightarrow g Z$, the Hamiltonian is covariant since $G$ and $B$ are coset parameters of $\mathrm{O}(d, d) / \mathrm{O}(d) \times \mathrm{O}(d)$ : $\mathcal{M}(G, B) \rightarrow \mathcal{M}^{\prime}=g^{T} \mathcal{M} g=\mathcal{M}\left(G^{\prime}, B^{\prime}\right)$ and so $\mathcal{H}_{\perp}(G, B) \rightarrow \mathcal{H}_{\perp}\left(G^{\prime}, B^{\prime}\right)$.

On the other hand the fundamental basis for a M2-brane are $Z_{M}=\left(p_{m}, \frac{1}{2} \epsilon^{i j} \partial_{i} x^{m} \partial_{i} x^{n}\right)$, $i=(1,2)$. Invariant symmetry of $\sigma^{i}$-diffeomorphism constraints, $\mathcal{H}_{i}=p_{m} \partial_{i} x^{m}=0$, is not apparent in this form. Multiplying $\epsilon^{i j} \partial_{j} x^{p}$ on $\mathcal{H}_{i}$ makes $\mathcal{H}^{p}$ to be bilinear in $Z_{m}$, although the metric $\tilde{\rho}^{M N ; p}$ is not manifestly duality symmetry invariant as seen in [23]. In this paper we pursue the diffeomorphism constraints in focusing on $d=4$ case leading to the $\operatorname{SL}(5)$ duality.

The organization of this paper is the following: In section 2 a canonical analysis of a M2-brane is presented in the basis of the generalized geometry. In section 3 we show that a Courant bracket for a M2 brane is calculated and generalized gauge transformation of $G_{m n}$ and $C_{m n l}$ are derived by using the Courant bracket. In section 4 we examine the invariance of the diffeomorphism constraints for $d=4$ case. We show that $\sigma^{i}$-diffeomorphism constraints are recast into a $\mathrm{SL}(5)$ vector in terms of the basis in a form of $\mathrm{SL}(5)$ rank two tensor, resulting SL(5) invariance on the constrained surface. The Hamiltonian constraint is also rewritten in terms of the tensor basis, and the generalized metric is rewritten as a rank four tensor, antisymmetric in a pair of two indices and symmetric in pairs, parameterized by $G_{m n}$ and $C_{m n l}$. We show that the generalized metric is written in a quartic form of the generalized vielbein given in $T^{4}$ compactified supergravity theory [26, 29, 30]. Then reformulated diffeomorphism constraints are manifestly SL(5) covariant in a gauge which depends on the background. It leads to SL(5) duality symmetry transformation for supergravity fields.

\section{Hamiltonian for a M2-brane}

We begin with an action for a M2-brane

$$
I=\int_{M} d^{3} \sigma\left(\mathcal{L}_{0}+\mathcal{L}_{W Z}\right)
$$




$$
\begin{aligned}
\mathcal{L}_{0} & =-T_{M 2} \sqrt{-h}, \quad h=\operatorname{det} h_{\mu \nu}, \quad h_{\mu \nu}=\partial_{\mu} x^{m} \partial_{\nu} x^{n} G_{m n} \\
\mathcal{L}_{W Z} & =\frac{1}{3 !} T_{M 2} \epsilon^{\mu \nu \rho} \partial_{\mu} x^{m} \partial_{\nu} x^{n} \partial_{\rho} x^{l} C_{m n l}
\end{aligned}
$$

where $G_{m n}(x)$ is the background metric and $C_{m n l}(x)$ is a rank three anti-symmetric gauge field. We focus on the bosonic part only throughout this paper. Target space indices, the 3-dimensional world volume indices and spatial world volume indices are denoted by $m, n, \cdots=0, \cdots, d-1 ; \mu, \nu, \cdots=0,1,2$ and $i, j, \cdots=1,2$ respectively. The canonical momenta are defined as

$$
p_{m}=T_{M 2}\left(-\sqrt{-h} h^{0 \mu} G_{m n} \partial_{\mu} x^{n}+\frac{1}{2} \epsilon^{0 i j} \partial_{i} x^{n} \partial_{j} x^{l} C_{m n l}\right) .
$$

The Hamiltonian constraint and the $\sigma^{i}$-diffeomorphism constraints are

$$
\left\{\begin{array}{l}
\mathcal{H}_{\perp}=\frac{1}{2 T_{M 2}}\left(\tilde{p}_{m} G^{m n} \tilde{p}_{n}+T_{M 2}^{2} \operatorname{det} h_{i j}\right)=0 \\
\mathcal{H}_{i}=\partial_{i} x^{m} \tilde{p}_{m}=\partial_{i} x^{m} p_{m}=0
\end{array}\right.
$$

with

$$
\begin{aligned}
\tilde{p}_{m} & \equiv p_{m}-\frac{T_{M 2}}{2} \epsilon^{0 i j} \partial_{i} x^{n} \partial_{j} x^{l} C_{m n l} \\
& =-T_{M 2} \sqrt{-h} h^{\mu 0} G_{m n} \partial_{\mu} x^{n},
\end{aligned}
$$

similar to the IIA D2 brane case [31]. The determinant term can be rewritten as

$$
\operatorname{det} h_{i j}=\frac{1}{2}\left(\epsilon^{i j} \partial_{i} x^{m} \partial_{j} x^{n}\right) G_{m m^{\prime}} G_{n n^{\prime}}\left(\epsilon^{i^{\prime} j^{\prime}} \partial_{i^{\prime}} x^{m^{\prime}} \partial_{j^{\prime}} x^{n^{\prime}}\right) .
$$

For simplicity we take a unit $T_{M 2}=1$ throughout the rest of this paper. Then the Hamiltonian constraint for the M2-brane in curved background is given by the $Z_{M}$ basis and the generalized metric as

$$
\begin{aligned}
& \mathcal{H}_{\perp}= \frac{1}{2} Z_{M} \mathcal{M}^{M N} Z_{N} \\
& Z_{N}=\left(\begin{array}{c}
p_{n} \\
\frac{1}{2} \epsilon^{i j} \partial_{i} x^{n} \partial_{j} x^{n^{\prime}}
\end{array}\right) \\
& \mathcal{M}^{M N}=\left(\begin{array}{cc}
G^{m n} & -G^{m k} C_{k n n^{\prime}} \\
-C_{m m^{\prime} l} G^{l n} & G_{[m \mid n} G_{\left.\mid m^{\prime}\right] n^{\prime}}+C_{m m^{\prime} l} G^{l k} C_{k n n^{\prime}}
\end{array}\right) .
\end{aligned}
$$

It is rewritten as

$$
\begin{aligned}
\mathcal{M}^{M N} & =\left(\mathcal{N}^{T}\right)^{M}{ }_{L} \mathcal{M}_{0}{ }^{L K} \mathcal{N}_{K}{ }^{N} \\
\mathcal{M}_{0}^{L K} & =\left(\begin{array}{cc}
G^{l k} & 0 \\
0 & G_{[l \mid k} G_{\left[l^{\prime}\right] k^{\prime}}
\end{array}\right), \mathcal{N}_{K}{ }^{N}=\left(\begin{array}{cc}
\delta_{k}{ }^{n} & -C_{k n n^{\prime}} \\
0 & \delta_{n}^{k} \delta_{n^{\prime}}^{k^{\prime}}
\end{array}\right) .
\end{aligned}
$$


It is further rewritten as

$$
\begin{aligned}
& \mathcal{M}_{0}^{M N}=\left(\mu^{T}\right)^{M}{ }_{A} \eta^{A B} \mu_{B}{ }^{N}, \quad \eta^{A B}=\left(\begin{array}{cc}
\delta^{a b} & 0 \\
0 & \delta_{[a \mid b} \delta_{\left.\mid a^{\prime}\right] b^{\prime}}
\end{array}\right), \mu_{A}{ }^{K}=\left(\begin{array}{cc}
e_{a}{ }^{k} & 0 \\
0 & \frac{1}{2} e_{[k}{ }^{a} e_{\left.k^{\prime}\right]} a^{\prime}
\end{array}\right) \\
& \mathcal{M}^{M N}=\left(\nu^{T}\right)^{M}{ }_{A} \eta^{A B} \nu_{B}{ }^{N}, \nu_{A}{ }^{N}=\mu_{A}{ }^{K} \mathcal{N}_{K}{ }^{N}=\left(\begin{array}{cc}
e_{a}{ }^{n} & -e_{a}{ }^{k} C_{k n n^{\prime}} \\
0 & \frac{1}{2} e_{[n}{ }^{a} e_{\left.n^{\prime}\right]} a^{\prime}
\end{array}\right),
\end{aligned}
$$

with $G_{m n}=e_{m}{ }^{a} e_{n}{ }^{b} \eta_{a b}$. The diffeomorphism constraints $\mathcal{H}_{i}(i=1,2)$ can be also written in terms of $Z_{M}$ basis by contracting with $\epsilon^{i j} \partial_{j} x^{p}$

$$
\mathcal{H}^{p} \equiv \frac{1}{2} \epsilon^{i j} \partial_{i} x^{p} \mathcal{H}_{j}=\frac{1}{2} Z_{M} \tilde{\rho}^{M N: p} Z_{N}=0 \quad, \quad \tilde{\rho}^{M N ; p}=\left(\begin{array}{cc}
0 & \delta_{[m}^{p} \delta_{l]}^{m} \\
\delta_{[m}^{p} \delta_{l]}^{n} & 0
\end{array}\right)
$$

where $\mathcal{H}^{p}(p=0, \ldots, d)$ are reducible.

Now $Z_{M}$ is a fundamental basis of the canonical analysis. The $Z_{M}$ algebra is given by

$$
\left\{Z_{M}(\sigma), Z_{N}\left(\sigma^{\prime}\right)\right\}=i \rho_{M N}^{i} \partial_{i} \delta\left(\sigma-\sigma^{\prime}\right), \quad \rho_{M N}^{i}=\left(\begin{array}{cc}
0 & \frac{1}{2} \epsilon^{i j} \partial_{j} x^{[n} \delta_{m}^{l]} \\
\frac{1}{2} \epsilon^{i j} \partial_{j} x^{[m} \delta_{n}^{l]} & 0
\end{array}\right)
$$

where $\rho_{M N}^{i}$ is divergenceless, $\partial_{i} \rho_{M N}^{i}=0$. The metric $\rho_{M N}^{i}$ in (2.6) reduces to the metric $\tilde{\rho}^{M N ; p}$ in (2.5) for the ground state in static gauge, $\partial_{j} x^{p}=\delta_{j}^{p}$.

\section{Courant bracket for M2 brane}

In this section we will write down a Courant bracket for a M2 brane explicitly in the notation of [23]. Let us consider a space generated by the algebra in (2.6). A vector in the space is given by

$$
\begin{gathered}
\hat{\Lambda}=\Lambda^{M} Z_{M}=\lambda+\lambda^{[2]} \in T \oplus \Lambda^{2} T^{*} \\
\lambda=\Lambda^{m} p_{m} \quad, \quad \lambda^{[2]}=\frac{1}{2} \Lambda_{m n} \frac{1}{2} \epsilon^{i j} \partial_{i} x^{m} \partial_{j} x^{n} .
\end{gathered}
$$

The canonical commutator between two vectors $\hat{\Lambda}_{1}(\sigma)$ and $\hat{\Lambda}_{2}\left(\sigma^{\prime}\right)$ is given by

$$
\left\{\hat{\Lambda}_{1}(\sigma), \hat{\Lambda}_{2}\left(\sigma^{\prime}\right)\right\}=-i \hat{\Lambda}_{12}(\sigma) \delta\left(\sigma-\sigma^{\prime}\right)+i\left(\frac{1-K}{2} \Psi_{(12)}^{i}(\sigma)-\frac{1+K}{2} \Psi_{(12)}^{i}\left(\sigma^{\prime}\right)\right) \partial_{i} \delta\left(\sigma-\sigma^{\prime}\right)
$$

with

$$
\hat{\Lambda}_{12}=\Lambda_{[1}^{l} \partial_{l} \Lambda_{2]}^{m} p_{m}
$$




$$
\begin{aligned}
& +\frac{1}{2}\left(\mathcal{L}_{\lambda_{[1}} \lambda_{2]}^{[2]}-\partial_{[m \mid}\left(\Lambda_{[1}^{l} \Lambda_{2] l \mid n]}\right)+K \partial_{[m \mid}\left(\Lambda_{(1}{ }^{l} \Lambda_{2) l \mid n]}\right)\right) \frac{1}{2} \epsilon^{i j} \partial_{i} x^{m} \partial_{j} x^{n} \\
\Psi_{(12)}^{i}= & \Lambda_{(1}{ }^{m} \Lambda_{2) m n} \frac{1}{2} \epsilon^{i j} \partial_{j} x^{n}=\frac{1}{2} \Lambda_{1}{ }^{M} \Lambda_{2}{ }^{N} \rho_{M N}^{i} \\
\mathcal{L}_{\lambda_{[1}} \lambda_{2]}^{[2]}= & \frac{1}{2}\left(\Lambda_{[1}^{l} \partial_{l} \Lambda_{2] m n}+\partial_{[m \mid} \Lambda_{[1}^{l} \Lambda_{2] l \mid n]}\right) \frac{1}{2} \epsilon^{i j} \partial_{i} x^{m} \partial_{j} x^{n} .
\end{aligned}
$$

$K$ is an arbitrary constant reflected by an ambiguity of $\partial_{i} \delta\left(\sigma-\sigma^{\prime}\right)$ as shown in [23]. Courant bracket for a M2-brane is given as $\hat{\Lambda}_{12}$ part in (3.2);

$$
\begin{gathered}
{\left[\hat{\Lambda}_{1}, \hat{\Lambda}_{2}\right]_{M 2}=\left[\lambda_{1}, \lambda_{2}\right]+\mathcal{L}_{\lambda_{[1} \lambda_{2]}^{[2]}-d\left(\iota_{\lambda_{[1}} \lambda_{2]}^{[2]}\right)}} \\
\left\{\begin{array}{c}
{\left[\lambda_{1}, \lambda_{2}\right]=\Lambda_{[1}^{n} \partial_{n} \Lambda_{2]}^{m} p_{m}} \\
\mathcal{L}_{\lambda_{[1} \lambda_{2]}^{[2]}}=\frac{1}{2}\left(\Lambda_{[1}^{l} \partial_{l} \Lambda_{2] m n}+\partial_{[m \mid} \Lambda_{[1}^{l} \Lambda_{2] l \mid n]}\right) \frac{1}{2} \epsilon^{i j} \partial_{i} x^{m} \partial_{j} x^{n} \\
d\left(\iota_{\lambda_{[1}} \lambda_{2]}^{[2]}\right)=\frac{1}{2} \partial_{[m \mid}\left(\Lambda_{[1}^{l} \Lambda_{2] l \mid n]}\right) \frac{1}{2} \epsilon^{i j} \partial_{i} x^{m} \partial_{j} x^{n}
\end{array}\right.
\end{gathered}
$$

for $K=0$, and

$$
\begin{gathered}
{\left[\hat{\Lambda}_{1}, \hat{\Lambda}_{2}\right]_{M 2}=\left[\lambda_{1}, \lambda_{2}\right]+\mathcal{L}_{\lambda_{1}} \lambda_{2}^{[2]}-\iota_{\lambda_{2}} d \lambda_{1}^{[2]}} \\
\left\{\begin{array}{l}
\mathcal{L}_{\lambda_{1}} \lambda_{2}^{[2]}=\frac{1}{2}\left(\Lambda_{1}^{l} \partial_{l} \Lambda_{2 m n}+\partial_{[m \mid} \Lambda_{1}^{l} \Lambda_{2 l \mid n]}\right) \frac{1}{2} \epsilon^{i j} \partial_{i} x^{m} \partial_{j} x^{n} \\
\iota_{\lambda_{2}} d \lambda_{1}^{[2]}=\Lambda_{2}^{l} \partial_{[l \mid} \Lambda_{1 \mid m n]}^{[2]} \epsilon^{i j} \partial_{i} x^{m} \partial_{j} x^{n}
\end{array}\right.
\end{gathered}
$$

for $K=1$.

Now let us calculate the generalized gauge transformation. It is convenient to introduce

$$
\left(\hat{C}_{a}\right)^{M}=\left(\begin{array}{c}
e_{a}{ }^{m} \\
e_{a}{ }^{l} C_{m n l}
\end{array}\right), e_{m}{ }^{a} e_{a}^{n}=\delta_{m}^{n}
$$

where $a$ is the local $\mathrm{SO}(d)$ index. The gauge transformation rule is given by the Courant bracket in (3.5) as

$$
\delta_{\xi}\left(\hat{C}_{a}\right)^{M}=\left[\hat{\xi},\left(\hat{C}_{a}\right)^{M}\right]_{M 2} \quad, \quad \hat{\xi}=\left(\begin{array}{c}
\xi^{m} \\
\xi_{m n}^{[2]}
\end{array}\right) .
$$

From the above transformation we obtain expected transformations of $G$ and $C$ by contracted the local $\mathrm{SO}(d)$ indices,

$$
\left\{\begin{array}{l}
\delta_{\xi} G_{m n}=\xi^{l} \partial_{l} G_{m n}+\partial_{(m \mid} \xi^{l} G_{l \mid n)} \\
\left.\delta_{\xi} C_{m n l}=\xi^{p} \partial_{p} C_{m n l}+\partial_{[m \mid} \xi^{p} C_{p \mid n l]}+\partial_{[m} \xi^{[2]} n l\right]
\end{array}\right.
$$

It turns out that the gauge parameter $\xi^{[2]}{ }_{m n}$ has a further gauge invariance, namely gauge symmetry of gauge symmetry. It is given by the invariance of the Courant bracket. 
We assume that $\Lambda^{M}$ 's are functions of only $x^{m}$. Under local transformations, $\delta \hat{\Lambda}_{i}, i=1,2$, invariance of the Courant bracket up to the total derivative is given as

$$
\delta \hat{\Lambda}_{i} \Rightarrow \delta \int d^{2} \sigma\left[\hat{\Lambda}_{1}, \hat{\Lambda}_{2}\right]_{M 2}(\sigma)=0
$$

There is no further gauge symmetry of $\Lambda_{i}{ }^{m}$ as seen from the coefficient of $p_{m}$ in (3.9). But there exists gauge symmetry of the parameter $\Lambda_{i ; m n}^{[2]}$ as seen as below; The coefficient of $\frac{1}{2} \epsilon^{i j} \partial_{i} x^{m} \partial_{j} x^{n}$ in (3.9) for $K=1$ allows further gauge invariance as

$$
\begin{aligned}
\delta \Lambda_{i ; m n}^{[2]}=\partial_{[m \mid} \zeta_{i \mid n]} \Rightarrow \delta\left(\mathcal{L}_{\lambda_{1}} \lambda_{2}^{[2]}-\iota \lambda_{2} d_{\lambda_{2}} d \lambda_{1}^{[2]}\right) & =\partial_{m}\left(\Lambda_{1}^{l} \partial_{[l} \zeta_{2 \mid n]}\right) \frac{1}{2} \epsilon^{i j} \partial_{i} x^{m} \partial_{j} x^{n} \\
& =\partial_{i}\left(\Lambda_{1}^{l} \partial_{[l} \zeta_{2 \mid n]} \frac{1}{2} \epsilon^{i j} x^{m} \partial_{j} x^{n}\right)
\end{aligned}
$$

Therefore gauge symmetry of the gauge parameter is given as

$$
\delta \Lambda_{i}^{M}=\left\{\begin{aligned}
\delta \Lambda_{i}{ }^{m} & =0 \\
\delta \Lambda_{i m n}{ }^{[2]} & =\partial_{[m \mid} \zeta_{i \mid n]}
\end{aligned}\right.
$$

When the parameter is chosen as $\zeta_{i ; n}=c_{n} \zeta_{i}$ with a constant vector $c_{m}$, the transformation becomes $\delta \Lambda_{i}=-c_{[m} \partial_{n]} \zeta_{i}$ as the survived component of $c_{l} \tilde{\rho}^{M N ; l} \partial_{N} \zeta_{i}$. The gauge symmetry of the gauge parameter $\hat{\xi}$ in $(3.8)$ is $\delta \xi^{m}=0, \quad \delta \xi_{m n}^{[2]}=\partial_{[m} \zeta_{n]}$.

\section{$4 \quad \mathrm{SL}(5)$ duality}

Diffeomorphism constraint $\mathcal{H}_{\sigma}$ for a bosonic string theory is invariant under T-duality symmetry. However this is not apparent for a membrane theory. The duality symmetry of the background field should be a reflection of the duality symmetry of the world volume mechanics of $Z_{M}(\sigma)$. So diffeomorphism constriants for a M2-brane should have U-duality symmetry.

The pure gravity theory has global $\mathrm{G}=\mathrm{SL}(d)$ and local $\mathrm{H}=\mathrm{SO}(d)$ symmetries, where the vielbein field is an element of the coset $\mathrm{G} / \mathrm{H} ; e_{m}{ }^{a} \rightarrow g_{m}{ }^{n} e_{n}{ }^{b} h_{b}{ }^{a}$ with $\mathrm{G} \ni g$ and $\mathrm{H} \ni h$. Including to the shift of the dilatation field, GL(1), it is extended to the hidden symmetry which is larger symmetry than the manifest $d$-dimensional invariance with subgroup $\mathrm{SL}(d) \times \mathrm{GL}(1)[26]$. In this section we focus on $d=4$ case and compare it with the known result of the SL(5) hidden symmetry of the supergravity theory. We analyze the symmetry of $\sigma^{i}$-diffeomorphism constraints for a M2-brane at first. Then we obtain the duality transformation for $G_{m n}$ and $C_{m n l}$ from the SL(5) transformation of the Hamiltonian for a M2 brane coupled to supergravity background, which is compared with the U-duality transformation of the $T^{4}$ reduced supergravity theory. At the same time we reformulate the diffeomorphism constraints and the Hamiltonian constraint in a manifest SL(5) symmetric way. 


\subsection{SL(5) invariance of M2 diffeomorphism constraints}

Let us begin with the $\sigma^{i}$-diffeomorphism constraints in $(2.5), \mathcal{H}^{p}=Z_{M} \tilde{\rho}^{M N ; p} Z_{N}=0$, which would have larger symmetry than the manifest $d$-dimensional invariance. The number of basis $Z_{M}$ is $d+\frac{1}{2} d(d-1)=\frac{1}{2} d(d+1)$, which is the number of the antisymmetric rank two tensor in $(d+1)$-dimensions. A question is whether the diffeomorphism constraint manifests the U-duality symmetry as was the case of string where the T-duality is realized as the $\mathrm{O}(d, d)$ invariance of the metric $\rho^{M N}$ in (1.1).

In 4-dimensions the basis $Z_{M}$ is identified as $10=4+6$-dimensional representation of $\mathrm{SL}(5)$. It is rewritten as a 5-dimensional rank two anti-symmetric tensor as

$$
Z_{M}=\left(\begin{array}{c}
p_{m} \\
\frac{1}{2} \epsilon^{i j} \partial_{i} x^{m} \partial_{j} x^{n}
\end{array}\right) \Rightarrow \check{Z}_{\hat{m} \hat{n}}=\left\{\begin{array}{c}
\check{Z}_{\sharp n}=p_{n} \\
\check{Z}_{m n}=\frac{1}{2} \epsilon_{m n p q} \epsilon^{i j} \partial_{i} x^{p} \partial_{j} x^{q} \\
\hat{m}=(\sharp, m) \text { and } m=1, \cdots, 4 .
\end{array}\right.
$$

Similar representation have been used in [32]. Under infinitesimal SL(5) transformations

$$
A_{\hat{m}}^{\hat{n}}=\left(\begin{array}{cc}
-\hat{\alpha} & \gamma^{n} \\
\beta_{m} & \alpha_{m}{ }^{n}
\end{array}\right) \quad, \quad \hat{\alpha}=\alpha_{m}{ }^{m},
$$

covariant and contravariant vectors are transformed linearly as $\delta u_{\hat{m}}=A_{\hat{m}}{ }^{\hat{n}} u_{\hat{n}}, \delta v^{\hat{m}}=-$ $v^{\hat{n}} A_{\hat{m}}^{\hat{n}}$, so that $\left(v^{\hat{m}} u_{\hat{m}}\right)$ is invariant. Thus the rank two tensor $\check{Z}_{\hat{m} \hat{n}}=\left(\check{Z}_{\sharp n}, \check{Z}_{m n}\right)$ in $(4.1)$ transforms as

$$
\begin{aligned}
\delta \check{Z}_{\hat{m} \hat{n}} & =A_{[\hat{m} \mid} \check{Z}_{\check{Z}_{\hat{l} \mid \hat{n}]}} \\
& =\left\{\begin{array}{l}
\delta \check{Z}_{\sharp n}=-\hat{\alpha} \check{Z}_{\sharp n}+\alpha_{n}{ }^{l} \check{Z}_{\sharp l}+\gamma^{l} \check{Z}_{l n} \\
\delta \check{Z}_{m n}=\beta_{[m \mid} \check{Z}_{\sharp \mid n]}+\alpha_{[m}{ }^{l} \check{Z}_{l \mid n]}
\end{array} .\right.
\end{aligned}
$$

In this basis the $\sigma^{i}$-diffeomorphism constraint in (2.5) is written as

$$
\mathcal{H}^{p}=\frac{1}{2} Z_{M} \tilde{\rho}^{M N ; p} Z_{N}=\epsilon^{m n l p} \check{Z}_{\sharp m} \check{Z}_{n l}=0 .
$$

Under SL(5) in (4.3) the diffeomorphism constraints are invariant as

$$
\delta \mathcal{H}^{m}=-\mathcal{H}^{n} \alpha_{n}{ }^{m}=0
$$

Noting $\check{Z}_{m n}=\epsilon_{m n p q} \frac{1}{2} \epsilon^{i j} \partial_{i} x^{p} \partial_{j} x^{q}$ in (4.1) it holds an identity

$$
\mathcal{H}^{\sharp} \equiv \epsilon^{m n l p} \check{Z}_{m n} \check{Z}_{l p}=0
$$

It leads to the diffeomorphism constraints as a SL(5) vector form,

$$
\mathcal{H}^{\hat{m}}=\frac{1}{8} \epsilon^{\hat{m} \hat{n} \hat{l} \hat{p} \hat{q}} \check{Z}_{\hat{n} \hat{l}} \check{Z}_{\hat{p} \hat{q}}, \hat{m}=(\sharp, m) .
$$


Under the SL(5) transformation it is transformed as

$$
\begin{aligned}
& \check{Z}_{\hat{m} \hat{n}} \rightarrow g_{\hat{m}}^{\hat{m}^{\prime}} g_{\hat{n}}^{\hat{n}^{\prime}} \check{Z}_{\hat{m}^{\prime} \hat{n}^{\prime}}, \quad g=1+A \in \mathrm{SL}(5)
\end{aligned}
$$

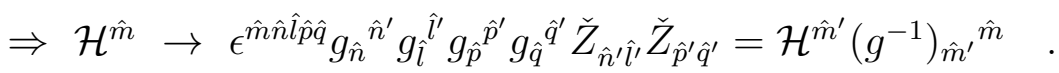

It is mentioned that $\mathrm{O}(3,3)$ symmetry can be seen from the metric of the $\sigma^{i}$-diffeomorphism constraint $\tilde{\rho}^{M N ; p}$ in (2.5). If we choose the direction of $\mathcal{H}^{p}$ vector to be $\mathcal{H}^{1}$ by the $\operatorname{SO}(d)$ rotation, then off-diagonal element $\delta_{[n l]}^{1 m}$ becomes $\delta_{l}^{m}$ for $l=2, \cdots, d$, resulting its rank to be $(d-1)$. Using an elementary matrix $\mathcal{P}$ it is written as

$$
\tilde{\rho}^{M N ; p}=\mathcal{P}^{T}\left(\begin{array}{cc|c}
0 & \mathbf{1}_{(d-1)} & 0 \\
\mathbf{1}_{(d-1)} & 0 & 0 \\
\hline 0 & 0 & 0
\end{array}\right) \mathcal{P}
$$

which contain $\mathrm{O}(d-1, d-1)$ invariance manifestly. This gauge choice may be related to the double field formalism. The more general argument on the relation between M theory duality basis and double field theory basis is given in [16].

\subsection{SL(5) duality transformation from M2 Hamiltonian}

It is known that there exists a global symmetry in $d$-dimensionally reduced supergravity theory which is larger than the one for the pure gravity theory, $\operatorname{SL}(d, \mathbf{R}) \times \operatorname{GL}(1, \mathbf{R})$. For the supergravity theory with $T^{4}$ it is $\operatorname{SL}(5, \mathbf{R})$ including the subgroup $\operatorname{SL}(4, \mathbf{R}) \times \operatorname{GL}(1, \mathbf{R})$.

The gauge field $\left(\hat{C}_{a}\right)^{M}$ in (3.6) is recasted in the $\check{Z}_{\hat{m} \hat{n}}$ basis in (4.1) as

$$
\left(\hat{C}_{a}\right)^{M} Z_{M}=\frac{1}{2}\left(\check{C}_{a}\right)^{\hat{m} \hat{n}} Z_{\hat{m} \hat{n}}, \quad\left(\check{C}_{a}\right)^{\hat{m} \hat{n}}=\left\{\begin{array}{rl}
\left(\check{C}_{a}\right)^{\sharp n} & =e_{a}^{n} \\
\left(\check{C}_{a}\right)^{m n} & =\frac{1}{2} \epsilon^{m n p q}\left(\hat{C}_{a}\right)_{p q}
\end{array},\right.
$$

which is also written as

$$
\left(\check{C}_{a}\right)^{m n}=\frac{1}{2} \epsilon^{n p q m} e_{a}^{l} C_{p q l}=\tilde{C}^{[m} e_{a}^{n]}, \quad \tilde{C}^{m}=\frac{1}{3 !} \epsilon^{m n l p} C_{n l p} .
$$

The Hamiltonian constraint for a membrane in (2.3) is written as

$$
\begin{aligned}
\mathcal{H}_{\perp} & =\frac{1}{2} Z_{M} \mathcal{M}^{M N} Z_{N}=\frac{1}{8} \check{Z}_{\hat{m} \hat{n}} \check{\mathcal{M}}^{\hat{m} \hat{n} ; \hat{p} \hat{q}} \check{Z}_{\hat{p} \hat{q}} \\
\check{\mathcal{M}}^{\hat{m} \hat{n} ; \hat{p} \hat{q}} & =\frac{1}{4} W_{\hat{a} \hat{b}}^{\hat{m} \hat{n}} \eta^{\hat{a} \hat{b} ; \hat{c} \hat{d}} W_{\hat{c} \hat{d}}^{\hat{p} \hat{q}}, \quad \eta^{\hat{a} \hat{b} ; \hat{c} \hat{d}}=\delta^{[\hat{a} \mid \hat{c}} \delta^{\mid \hat{b}] \hat{d}} \\
W_{\hat{a} \hat{b}}^{\hat{m} \hat{n}} & =\left(\begin{array}{cc}
e_{b}{ }^{n} & -\tilde{C}^{[m} e_{b}{ }^{n]} \\
0 & \mathbf{e} e_{a}^{[m} e_{b}{ }^{n]}
\end{array}\right),
\end{aligned}
$$


with indices $(\hat{a} \hat{b})=(\llbracket b, a b),(\hat{m} \hat{n})=(\sharp n, m n)$ and $\mathbf{e}=\operatorname{det} e_{m}{ }^{a} . W_{\hat{a} \hat{b}}^{\hat{m} \hat{n}}$ is a $10 \times 10$ matrix representation of $\nu_{A}{ }^{N}$ in (2.4). It is a coset element of $\mathrm{SL}(5) / \mathrm{SO}(5)$ expressed by $24-10=$ $10+4=14$ coset parameters $G_{m n}, C_{m n l}$. The $10 \times 10$ matrix $W_{\hat{a} \hat{b}}^{\hat{m} \hat{n}}$ contains the $4 \times 10$ matrix $\left(\check{C}_{a}\right)^{\hat{m} \hat{n}}$ manifestly and the local SO(5) symmetry is used for the triangular gauge.

Furthermore $W$ in (4.12) is a tensor product of the $5 \times 5$ representation of the coset $\mathrm{SL}(5) / \mathrm{SO}(5)$. It is known that the "generalized vielbein" for $T^{4}$ reduced supergravity as a coset element $\mathrm{SL}(5) / \mathrm{SO}(5)$ derived in $[26,29,30]$

$$
V_{\hat{m}}^{\hat{a}}=\left(\begin{array}{cc}
\mathbf{e}^{3 / 5} & \mathbf{e}^{-2 / 5} \tilde{C}^{l} e_{l}^{a} \\
0 & \mathbf{e}^{-2 / 5} e_{m}{ }^{a}
\end{array}\right), \quad \operatorname{det} V=1 .
$$

The SL(5) invariant current is constructed as $V^{-1} \partial_{\mu} V$. Bilinear of its coset part gives the $d$-dimensional part of supergravity action. It is transformed as $V \rightarrow g V h$ with $g \in \operatorname{SL}(5)$ and $h \in \mathrm{SO}(5)$. After the $\mathrm{SO}(5)$ pull back, the $\mathrm{SL}(5)$ transformation rules are given by

$$
\begin{aligned}
& \delta V_{\hat{m}}{ }^{\hat{a}}=A_{\hat{m}}{ }^{\hat{n}} V_{\hat{n}}{ }^{\hat{a}}+V_{\hat{m}}{ }^{\hat{b}} \lambda_{\hat{b}}{ }^{\hat{a}} \\
& \delta e_{m}{ }^{a}=\alpha_{m}{ }^{n} e_{n}{ }^{a}+\beta_{m} \tilde{C}^{n} e_{n}{ }^{a}+e_{m}{ }^{b} \lambda_{b}{ }^{a}-\frac{2}{3}\left(\hat{\alpha}+\tilde{C}^{l} \beta_{l}\right) e_{m}{ }^{a} \\
& \left\{\begin{array}{c}
\delta G_{m n}=\alpha_{(m \mid}{ }^{l} G_{l \mid n)}+\beta_{(m \mid} \tilde{C}^{l} G_{l \mid n)}-\frac{4}{3}\left(\hat{\alpha}+\tilde{C}^{l} \beta_{l}\right) G_{m n} \\
\delta \tilde{C}^{m}=-\hat{\alpha} \tilde{C}^{m}-\tilde{C}^{n} \alpha_{n}{ }^{m}+\mathbf{e}^{2} \beta_{n} G^{n m}-\beta_{l} \tilde{C}^{l} \tilde{C}^{m}+\gamma^{m}
\end{array}\right.
\end{aligned}
$$

where $\mathrm{SO}(4)$ symmetry parameter is $\lambda_{b}{ }^{a}$.

The SL(5) tensor $W_{\hat{a} \hat{b}}^{\hat{m} \hat{n}}$ is rewritten by this "generalized vielbein" as

$$
W_{\hat{a} \hat{b}}^{\hat{m} \hat{n}}=\mathbf{e}^{1 / 5}\left(V^{-1}\right)_{\hat{a}}^{[\hat{m}}\left(V^{-1}\right)_{\hat{b}}^{\hat{n}]} \quad, \quad V^{-1}{ }_{\hat{a}}^{\hat{m}}=\left(\begin{array}{cc}
\mathbf{e}^{-3 / 5} & -\mathbf{e}^{-3 / 5} \tilde{C}^{m} \\
0 & \mathbf{e}^{2 / 5} e_{a}^{m}
\end{array}\right)
$$

then the "generalized metric" in (4.12) is rewritten as quartic in the generalized vielbein as

$$
\begin{aligned}
\check{\mathcal{M}}^{\hat{m} \hat{n} ; \hat{p} \hat{q}} & =\frac{\mathbf{e}^{2 / 5}}{4}\left(V^{-1}\right)_{\hat{a}}^{[\hat{m}}\left(V^{-1}\right)_{\hat{b}}^{\hat{n}]} \eta^{\hat{a} \hat{b} ; \hat{a}^{\prime} \hat{b}^{\prime}}\left(V^{-1}\right)_{\hat{a}^{\prime}}{ }^{\prime \hat{p}}\left(V^{-1}\right)_{\hat{b}^{\prime}}^{\hat{q}]} \\
& =\left(\begin{array}{cc}
G^{n q} & -\tilde{C}^{[p} G^{q] n} \\
-\tilde{C}^{[m} G^{n] q} & \mathbf{e}^{2} G^{p[m} G^{n] q}+\tilde{C}^{[m} G^{n][q} \tilde{C}^{p]}
\end{array}\right),(\hat{m} \hat{n})=(\sharp n, m n) .
\end{aligned}
$$

The Hamiltonian constraint for a M2-brane in (2.2) is now written as

$$
\left.\mathcal{H}_{\perp}=\frac{\mathbf{e}^{2 / 5}}{8} J_{\hat{a} \hat{b}} \eta^{\hat{a} \hat{b} ; \hat{a}^{\prime} \hat{b}^{\prime}} J_{\hat{a}^{\prime} \hat{b}^{\prime}}, \quad J_{\hat{a} \hat{b}}=\frac{1}{2}\left(V^{-1}\right)_{\hat{a}}^{[\hat{m}} \check{Z}_{\hat{m} \hat{n}}\left(V^{-1 T}\right)^{\hat{n}]}\right]_{\hat{b}}
$$

where the current $J_{\hat{a} \hat{b}}$ is manifestly $\mathrm{SL}(5)$ invariant and $\mathrm{SO}(5)$ invariant to guarantee the $\mathrm{SL}(5)$ covariance of the system. By choosing a gauge of the $\tau$-diffeomorphism invariance $\mathbf{h}=\mathbf{e}^{-2 / 5}$

$$
H=\int d^{2} \sigma \mathbf{h} \mathcal{H}_{\perp}, \mathbf{h}=\mathbf{e}^{-2 / 5} \Rightarrow H=\int d^{2} \sigma \frac{1}{8} J_{\hat{a} \hat{b}} \eta^{\hat{a} \hat{b} ; \hat{a}^{\prime} \hat{b}^{\prime}} J_{\hat{a}^{\prime} \hat{b}^{\prime}}
$$


then the Hamiltonian has manifest SL(5) invariance. ${ }^{1}$ The SL(5) duality transformation of $G_{m n}$ and $C_{m n l}$ are given in (4.14).

\section{Summary and discussion}

We have seen how the SL(5) duality in M-theory is derived from the M2-brane mechanics. A Courant bracket for a M2-brane is obtained to derive the generalized gauge transformation rules for $G_{m n}$ and $C_{m n l}$. It is natural to use the anti-symmetric rank two basis $\check{Z}_{\hat{m} \hat{n}}$ as 10-representation of SL(5). In this basis the diffeomorphism constraints are rewritten as a SL(5) vector showing the SL(5) invariance on the constraint surface. The Hamiltonian is written by the rank four generalized metric which is quartic in the generalized vielbein. The generalized vielbein is an element of the coset $\mathrm{SL}(5) / \mathrm{SO}(5)$ parameterized by $G_{m n}$ and $C_{m n l}$. Thus the SL(5) duality transformation of $G_{m n}$ and $C_{m n l}$ is obtained as that of the coset parameter. The diffeomorphism and Hamiltonian constraints in a background dependent gauge are invariant under the SL(5) transformations of the basis $\check{Z}_{\hat{m} \hat{n}} \rightarrow g_{\hat{m}}^{\hat{m}^{\prime}} g_{\hat{n}}^{\hat{n}^{\prime}} Z_{\hat{m}^{\prime} \hat{n}^{\prime}}$, when the coset parameters $G_{m n}, C_{m n l}$ are transformed in the non-linear realization of SL(5)/SO(5).

The extension of this analysis to M5-brane system involves 16-representation of $\mathrm{SO}(5,5)$ duality symmetry in $d=5$. The 16 -representation of $\mathrm{SO}(5,5)$ contains $5+10+1$ which corresponds to the usual vector, 2 -form and 5 -form in $d=5$. Including such higher dimensional cases manifestation of duality symmetries is forthcoming problem. Treatment of RR gauge fields and fermionic fields are also necessary to be clarified.

\section{Acknowledgements}

M.H. would like to thank Warren Siegel and Maxim Zabzine for fruitful discussions. She is also grateful to the 2012 Summer Simons workshop in Mathematics and Physics for a stimulating environment and its warm hospitality. The work of M.H. is supported by Grantin-Aid for Scientific Research (C) No. 24540284 from The Ministry of Education, Culture, Sports, Science and Technology of Japan.

\section{References}

[1] N. Hitchin, "Generalized Calabi-Yau manifolds," Quart. J. Math. Oxford Ser. 54 (2003) 281 [arXiv:math/0209099].

\footnotetext{
${ }^{1}$ This is related to the fact that dilaton field is required to construct the Ricci scalar from T-dual manifest covariant derivatives as shown in [5]. We thank Warren Siegel for explaining this. It is also mentioned that the enlargement of dimensions may be related to F-theory.
} 
[2] M. Gualtieri, "Generalized complex geometry," math/0401221 [math-dg].

[3] C. M. Hull, "Generalised Geometry for M-theory," JHEP 0707 (2007) 079 [hepth/0701203].

[4] W. Siegel, "Two vierbein formalism for string inspired axionic gravity," Phys. Rev. D 47 (1993) 5453 [hep-th/9302036].

[5] W. Siegel, "Superspace duality in low-energy superstrings," Phys. Rev. D 48 (1993) 2826 [hep-th/9305073]; "Manifest T-duality in low-energy superstrings," [arXiv:hepth/9308133].

[6] A. A. Tseytlin, "Duality Symmetric Formulation Of String World Sheet Dynamics," Phys. Lett. B 242 (1990) 163.

[7] C. M. Hull, " A geometry for non-geometric string backgrounds," JHEP 0510 (2005) 065 [hep-th/0406102];C. M. Hull and R. A. Reid-Edwards, "Flux compactifications of string theory on twisted tori," Fortsch. Phys. 57 (2009) 862 [hep-th/0503114].

[8] C. Hull and B. Zwiebach, 'Double Field Theory," JHEP 0909 (2009) 099 [arXiv:0904.4664 [hep-th]]; "The gauge algebra of double field theory and Courant brackets," JHEP 0909, 090 (2009) [arXiv:0908.1792 [hep-th]].

[9] G. Bonelli and M. Zabzine, "From current algebras for p-branes to topological Mtheory," JHEP 0509 (2005) 015 [hep-th/0507051];

Joel Ekstrand, Maxim Zabzine, "Courant-like brackets and loop spaces", JHEP 1103:074,2011, arXiv:0903.3215v3 [math-ph].

[10] P. P. Pacheco and D. Waldram, "M-theory, exceptional generalised geometry and superpotentials," JHEP 0809 (2008) 123 [arXiv:0804.1362 [hep-th]].

[11] M. Grana, J. Louis, A. Sim and D. Waldram, " $E_{7(7)}$ formulation of $\mathcal{N}=2$ backgrounds," JHEP 0907 (2009) 104 [arXiv:0904.2333 [hep-th]];

A. Coimbra, C. Strickland-Constable and D. Waldram, "Supergravity as Generalised Geometry I: Type II Theories," JHEP 1111 (2011) 091 [arXiv:1107.1733 [hep-th]]; " $E_{d(d)} \times \mathbf{R}^{+}$Generalised Geometry, Connections and M theory," arXiv:1112.3989 [hepth]; "Generalised Geometry and type II Supergravity," arXiv:1202.3170 [hep-th];

M. Grana and D. Marques, "Gauged Double Field Theory," arXiv:1201.2924 [hep-th].

[12] O. Hohm, C. Hull and B. Zwiebach, "Background independent action for double field theory," JHEP 1007 (2010) 016 [arXiv:1003.5027 [hep-th]]; "Generalized metric formulation of double field theory," JHEP 1008 (2010) 008 [arXiv:1006.4823 [hep-th]]; 
B. Zwiebach, "Double Field Theory, T-Duality, and Courant Brackets," arXiv:1109.1782 [hep-th].

[13] O. Hohm and S. K. Kwak, "Frame-like Geometry of Double Field Theory," J. Phys. A A 44 (2011) 085404 [arXiv:1011.4101 [hep-th]];

O. Hohm and B. Zwiebach, "On the Riemann Tensor in Double Field Theory," JHEP 1205 (2012) 126 [arXiv:1112.5296 [hep-th]]; "Large Gauge Transformations in Double Field Theory," arXiv:1207.4198 [hep-th].

[14] D. S. Berman and M. J. Perry, "Generalized Geometry and M-theory," JHEP 1106 (2011) 074 [arXiv:1008.1763 [hep-th]];

D. S. Berman, H. Godazgar and M. J. Perry, "SO $(5,5)$ duality in M-theory and generalized geometry," Phys. Lett. B 700 (2011) 65 [arXiv:1103.5733 [hep-th]];

D. S. Berman, H. Godazgar, M. Godazgar and M. J. Perry, "The Local symmetries of M-theory and their formulation in generalised geometry," JHEP 1201 (2012) 012 [arXiv:1110.3930 [hep-th]];

D. S. Berman, H. Godazgar, M. J. Perry and P. West, "Duality Invariant Actions and Generalised Geometry," JHEP 1202 (2012) 108 [arXiv:1111.0459 [hep-th]].

[15] I. Jeon, K. Lee and J. -H. Park, "Stringy differential geometry, beyond Riemann," Phys. Rev. D 84 (2011) 044022 [arXiv:1105.6294 [hep-th]]; "Incorporation of fermions into double field theory," JHEP 1111 (2011) 025 [arXiv:1109.2035 [hep-th]]; "Supersymmetric Double Field Theory: Stringy Reformulation of Supergravity," Phys. Rev. D 85 (2012) 081501 [arXiv:1112.0069 [hep-th]]; "Ramond-Ramond Cohomology and O(D,D) T-duality," arXiv:1206.3478 [hep-th].

[16] D. C. Thompson, "Duality Invariance: From M-theory to Double Field Theory," JHEP 1108 (2011) 125 [arXiv:1106.4036 [hep-th]].

[17] D. Andriot, M. Larfors, D. Lust and P. Patalong, "A ten-dimensional action for nongeometric fluxes," JHEP 1109 (2011) 134 [arXiv:1106.4015 [hep-th]].

[18] O. Hohm and S. K. Kwak, "N=1 Supersymmetric Double Field Theory," JHEP 1203 (2012) 080 [arXiv:1111.7293 [hep-th]].

[19] S. F. Hassan, "T duality, space-time spinors and RR fields in curved backgrounds," Nucl. Phys. B 568 (2000) 145 [hep-th/9907152]; "SO(d,d) transformations of RamondRamond fields and space-time spinors," Nucl. Phys. B 583 (2000) 431 [hep-th/9912236].

[20] P. Koerber, "Stable D-branes, calibrations and generalized Calabi-Yau geometry," JHEP 0508 (2005) 099 [hep-th/0506154]; "Lectures on Generalized Complex Geometry for Physicists," Fortsch. Phys. 59 (2011) 169 [arXiv:1006.1536 [hep-th]]; 
P. Koerber and L. Martucci, "Deformations of calibrated D-branes in flux generalized complex manifolds," JHEP 0612 (2006) 062 [hep-th/0610044].

[21] C. Albertsson, T. Kimura and R. A. Reid-Edwards, "D-branes and doubled geometry," JHEP 0904 (2009) 113 [arXiv:0806.1783 [hep-th]];

C. Albertsson, S. -H. Dai, P. -W. Kao and F. -L. Lin, "Double Field Theory for Double D-branes," JHEP 1109 (2011) 025 [arXiv:1107.0876 [hep-th]].

[22] O. Hohm, S. K. Kwak and B. Zwiebach, "Unification of Type II Strings and T-duality," Phys. Rev. Lett. 107 (2011) 171603 [arXiv:1106.5452 [hep-th]]; "Double Field Theory of Type II Strings," JHEP 1109 (2011) 013 [arXiv:1107.0008 [hep-th]].

[23] M. Hatsuda and T. Kimura, "Canonical approach to Courant brackets for D-branes," JHEP 1206 (2012) 034 [arXiv:1203.5499 [hep-th]].

[24] T. Kikuchi, T. Okada and Y. Sakatani, "Rotating string in doubled geometry with generalized isometries," arXiv:1205.5549 [hep-th];

T. Asakawa, S. Sasa and S. Watamura, "D-branes in Generalized Geometry and DiracBorn-Infeld Action," arXiv:1206.6964 [hep-th].

[25] C. M. Hull and P. K. Townsend, "Unity of superstring dualities," Nucl. Phys. B 438 (1995) 109 [hep-th/9410167].

[26] E. Cremmer and B. Julia, "The SO(8) Supergravity," Nucl. Phys. B 159 (1979) 141.

[27] M. J. Duff and J. X. Lu, "Duality Rotations In Membrane Theory," Nucl. Phys. B 347 (1990) 394;

M. J. Duff, "Duality Rotations In String Theory," Nucl. Phys. B 335 (1990) 610.

[28] M. K. Gaillard and B. Zumino, "Duality Rotations for Interacting Fields," Nucl. Phys. B 193 (1981) 221.

[29] E. Sezgin and A. Salam, "Maximal Extended Supergravity Theory In Sevendimensions," Phys. Lett. B 118 (1982) 359.

[30] Y. Tanii, "Supergravity," SGC library 82 (2011) Saiensu-sha (Japanese); "N=8 Supergravity In Six-dimensions," Phys. Lett. B 145 (1984) 197.

[31] M. Hatsuda and K. Kamimura, "Wess-Zumino actions for IIA D-branes and their supersymmetries," Nucl. Phys. B 535 (1998) 499 [hep-th/9804087].

[32] M. Cederwall, "M-branes on U-folds," arXiv:0712.4287 [hep-th]. 